\title{
Mitigating Hot Spot Problems in Wireless Sensor Networks Using Tier-Based Quantification Algorithm
}

\author{
R. Balamurali ${ }^{1}$, K. Kathiravan ${ }^{2}$ \\ ${ }^{1}$ IFHE (The ICFAI Foundation for Higher Education) University, Hyderabad, India \\ ${ }^{2}$ Easwari Engineering College, Ramapuram, Chennai, India \\ Emails:mohmur@gmail.com kathirraji@gmail.com
}

\begin{abstract}
One of the key issues in Wireless Sensor Networks (WSN) is the hot-spot problem i.e. the nodes closer to the sink tend to drain their energy at a faster rate when compared to other nodes as they have to perform more communication and hence the sensor network may get isolated. In this paper we are using an integrated $M A C$ and routing protocol which divides the network into tiers. We also propose a quantification algorithm, which decides the number of nodes in each tier to mitigate the hot-spot problem.
\end{abstract}

Keywords: WSN, hotspot problem, tier, quantification algorithm, sink.

\section{Introduction}

The advances of micro-sensing Micro-ElectroMechanical Systems (MEMS) and wireless communication technologies have promoted the development of wireless sensor networks. A WSN consists of many sensor nodes densely deployed in a field: individually able to collect environmental information and together able to support multihop ad hoc routing. WSNs provide an inexpensive and convenient way to monitor the physical environments. With their environment-sensing capability WSNs can enrich human life with their application in health care, building monitoring, and home security. Due to the advancement in MEMS and availability of low-cost sensor nodes lot of research is being conducted in Wireless Sensor Networks. The major issue, which most researches focus on, is energy consumption in WSN. One related issue is hot-spot problem.

\section{Problem definition}

WSN uses a multi-hop communication pattern i.e. the sensor nodes will not send the sensed parameter directly to the sink: rather they are passed on to the sink in a hop by hop fashion. So the nodes closer to the sink have to communicate their own sensed 
data and should also relay data from the other nodes. Hence they deplete their energy quicker than the other nodes. As a result the network may get isolated. In this paper we integrate MAC and Routing and form a tier-based architecture as proposed in [1]. Since it is generally assumed that sensor nodes would become inexpensive a simple solution to this problem is to add supplementary nodes in the hot-spot area [2]. We are also proposing a quantification algorithm, which decides the effective number of nodes in each tier to maximize the network life time and to reduce or to mitigate the hot-spot effect.

\section{Related work}

Several differing solutions are proposed to mitigate hot-spot effect as specified in [2-7]. A clustering approach, similar to LEACH, with varying cluster head is proposed in [3] to mitigate hot-spot affect. A multi-hop and hierarchical multi-hop routing approach is used in [4] to mitigate the hot-spot effect. An analysis of intelligent power control i.e. nodes far away from sink should transmit over a longer distance and data aggregation using an aggregator node to mitigate hot-spot effect is discussed in [5]. DEAR [6] algorithm optimizes each individual distance so that all sensor nodes consume their energy at a similar rate. Mobile sensors are used in [7] for surveillance. Besides mobile sensors nodes may self-configure as-well for mitigation of hot-spot effect.

The rest of the paper is divided into four parts: Section 4 - Tier-formation; Section 5 - Quantification algorithm; Section 6 - Simulation results; Section 7 Conclusion.

\section{Tier-formation}

To create tiers, we are following the approach proposed in [1]. We divide the nodes around the sink as tiers. The nodes closer to the sink (e.g., within a range of $250 \mathrm{~m}$ ) form tier1. The next level of nodes (e.g., which have a range of distance between 250 and $500 \mathrm{~m}$ ) form Tier 2. It follows the same calculation of the range of distance for the rest of the tiers. Tier formation consists of two phases: Setup phase and Action phase.

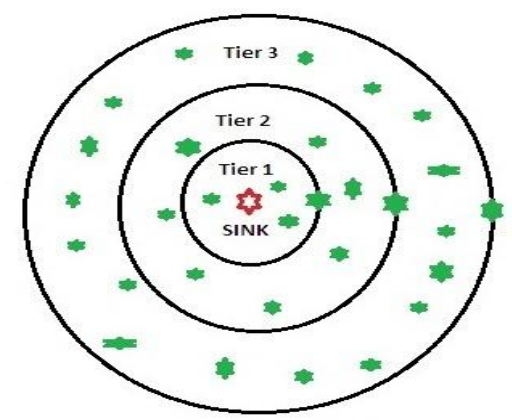

Fig. 1. Tier-formation 


\subsection{Setup phase}

Initially all nodes except the sink node's Tier ID will be set as -1 and the Tier ID of the sink will be 0 . The sink will broadcast a Hello Message with its Tier ID (i.e., 0), the nodes receiving this message will set their Tier ID as 1 (i.e., received Hello Message Tier ID +1 ). Now all these nodes with Tier ID 1 will broadcast the Hello Message. The nodes with Tier ID -1 receiving this hello message will update their Tier ID to 2 . This process continues until all nodes are assigned with a positive Tier ID. Thus the tier-formation will be completed at the end of the setup phase.

\subsection{Action phase}

If any node has to send a sensed data, the node will broadcast an RTS message with its Tier ID. The node in the previous tier receiving this RTS message (i.e., the Tier ID of the receiving node should be 2 to send a CTS if the sender's Tier ID is 3 ) can alone respond with a CTS message. Following this the sender node would send the Data packet to the node from which it first receives the CTS and the receiver node would send an acknowledgment to the sender. Now, the received node will broadcast the RTS and transmit the data packet to the next tier. This process continues until the data successfully reaches the sink.

The communication is commonly from node to sink and not from sink to node in WSN.

\section{Quantification algorithm}

Before, discussing the quantification algorithm, consider the following example.

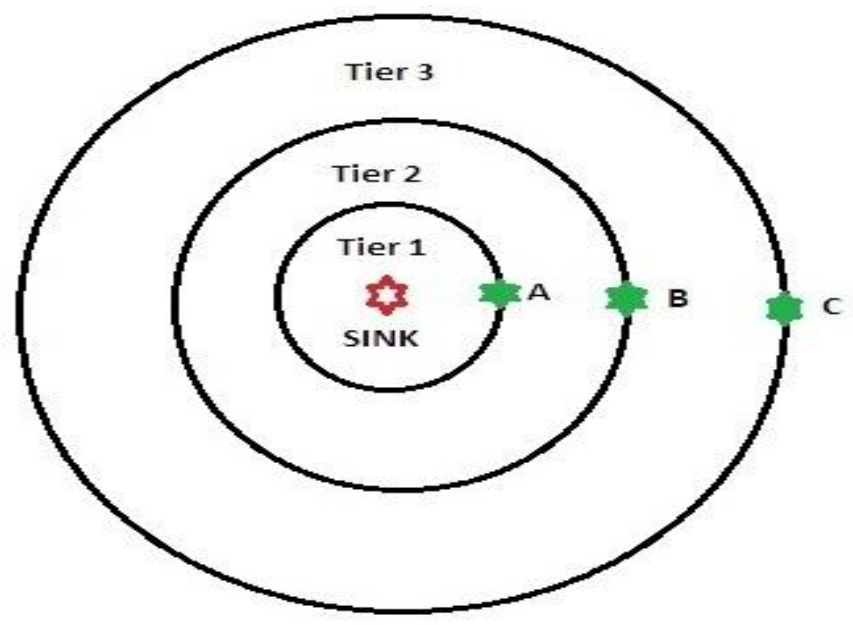

Fig. 2. Hotspot problem

In Fig. 2 there are three nodes A, B and C in the Tier 1, 2 and 3 respectively. Let us assume that all the three nodes have an initial energy level of $5 \mathrm{~J}$ and let us also assume that for every communication the node loses $1 \mathrm{~J}$ of energy. At a given instance of time, if all three nodes would like to communicate a sensed parameter to the sink, 
node $\mathrm{C}$ will communicate the sensed data to node $\mathrm{B}$ in concurrence with the tierbased multi-hop communication. Now node C's available energy will be $4 \mathrm{~J}$. Node B has to communicate its own data besides the data from $\mathrm{C}$, so its energy will become $3 \mathrm{~J}$ and node $\mathrm{A}$ has to communicate its own data besides communicating two data from B. So its energy will be $2 \mathrm{~J}$. At the end of Cycle 1, node A's energy will be $2 \mathrm{~J}$, node B's energy will be $3 \mathrm{~J}$ and node C's energy will be $4 \mathrm{~J}$.

Consider the same action for cycle 2, i.e., at a particular time instance, all three nodes would like to communicate the sensed parameter to the sink. Now, after C's communication, its energy will be $3 \mathrm{~J}$ and after $\mathrm{B}$ communicating its own sensed data and data from $\mathrm{C}$, its energy will be $1 \mathrm{~J}$ and A's available energy is only $2 \mathrm{~J}$, but it has to transmit 3 data, whereas it can communicate only 2 data and after that node A will be drained and as a result a hot-spot occurs and the network gets isolated.

We are proposing a very simple quantification algorithm by analyzing the above stated problem. The quantification algorithm is as follows.

If there are $x$ nodes in outer tier then there should be $2 x$ nodes in the next inner tier and $3 x$ nodes in the next inner tier and so on and there should be $n x$ nodes in the first tier, where $n$ is the total number of tiers.

Applying this quantification algorithm to the problem stated in Fig. 2, we will get a setup as it is shown in Fig. 3.

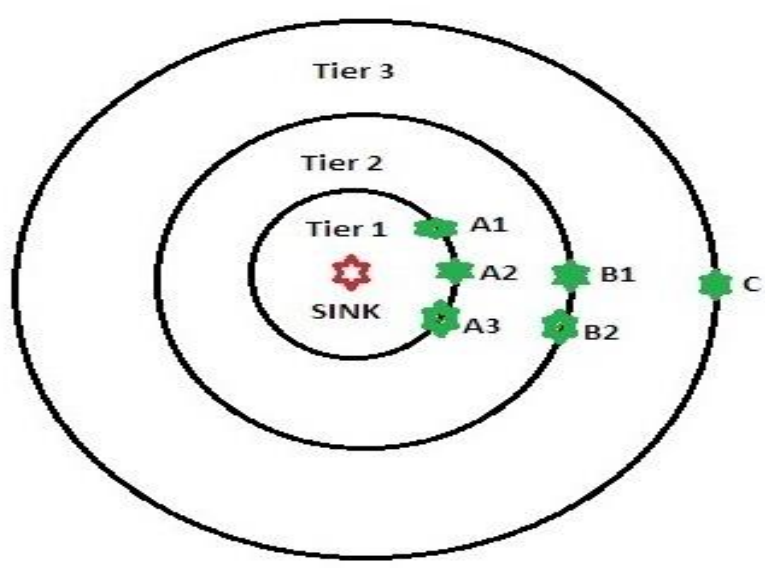

Fig. 3. Quantification algorithm based node allocation

Now, consider the situation that one node from each tier would like to communicate at a given instance of time and also let us assume that the initial energy of all nodes is $5 \mathrm{~J}$. Let us also assume that nodes B1 and B2 are in the same sensing range within Tier 2 and $\mathrm{A} 1, \mathrm{~A} 2$ and $\mathrm{A} 3$ are in the same sensing range within Tier 2 . Now during Cycle 1, node $\mathrm{C}$ is sending a sensed data to Tier 2 (let us assume to B1) and C's energy will become $4 \mathrm{~J}$. And, meanwhile, if any event occurs at Tier 2 then $\mathrm{B} 2$ will communicate that to Tier 1 as $\mathrm{B} 1$ is communicating with $\mathrm{C}$ and following which $\mathrm{B} 1$ will also communicate the data to Tier 1 . Now the energy of B1 and B2 will be $4 \mathrm{~J}$. Assuming that the data sent by $\mathrm{B} 1$ and $\mathrm{B} 2$ is received by $\mathrm{A} 1$ and $\mathrm{A} 2$ in Tier 1 respectively, then $\mathrm{A} 1$ and $\mathrm{A} 2$ will communicate this data to the sink. Meanwhile, if there is any event at Tier 1, this will be communicated by the node A3. So, by the end of Cycle 1 all nodes A1, A2, A3, B1, B2 and C have energy level of 
$4 \mathrm{~J}$. And if this continues, at the end of Cycle 5, all nodes energy will be 0 . It should be noted here that the events occur rarely in a WSN, what we have considered here is a worst-case scenario to produce a model for maximizing the life time of WSN. Compared to the basic model, proposed in Fig. 2, the model proposed in Fig. 3 increases the lifetime by four fold, which is a substantial improvement.

The quantification algorithm can be defined as follows, given the number of nodes $N$ :

$$
n x+(n-1) x+\ldots+2 x+1 x=N,
$$

where $x$ is the number of nodes in Tier $n$ and $n x$ is the number of nodes in Tier 1 .

\subsection{Benefit of the quantification algorithm}

The quantification algorithm tries to provide an approximate count on the number of nodes to be placed in each tier to reduce the impact of Hotspot. Instead of sensor nodes, if dedicated repeaters are used, then they should also be battery powered and further repeaters can't sense or compute. Hence, it is advantageous to use sensor nodes as repeaters rather than using dedicated repeaters.

As [2] suggests, a simple and efficient solution to mitigate the Hotspot problem is by adding additional nodes around the sink. Further, it is estimated that the cost of sensor nodes are getting cheaper and cheaper, hence adding more nodes to mitigate the hotspot effect and to increase the network lifetime is considered as the simple and efficient solution as of the current state-of-art.

\section{Simulation and experimental results}

We have made a Simulation with three scenarios, each having three tiers. In Scenario 1 (Fig. 4) there are 4 nodes in each tier, in Scenario 2 (Fig. 5) there are 8 nodes in Tier 1 and 4 nodes in other two tiers and in Scenario 3 (Fig. 6) there are 12 nodes in Tier 1, 8 nodes in the Tier 2 and 4 nodes in the last or Tier 3 . These three scenarios have been simulated with SMAC and Integrated MAC and routing algorithm. From simulation results it has been found that Tier based quantification gives better network life time for Integrated MAC and Routing algorithm than SMAC.

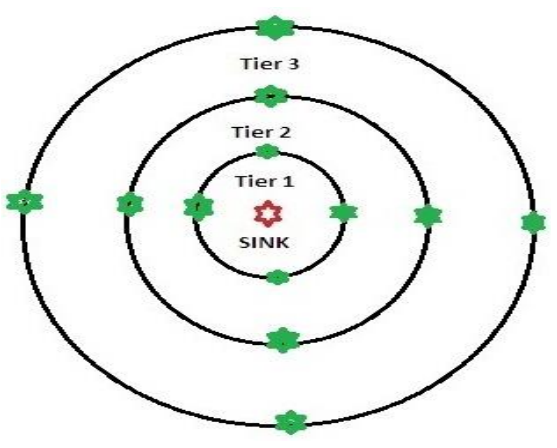

Fig. 4. Scenario 1

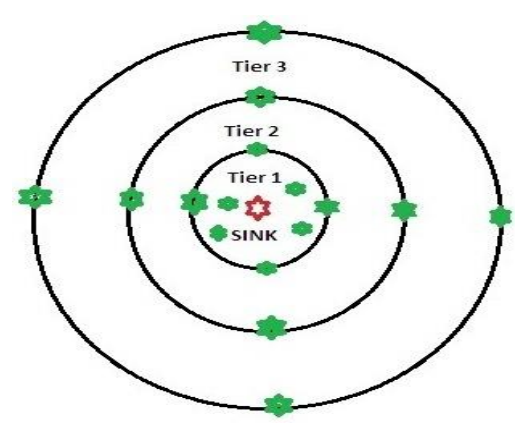

Fig. 5. Scenario 2 
From Fig. 7, we can observe a substantial improvement of overall lifetime of the network for Integrated MAC and Routing protocol.
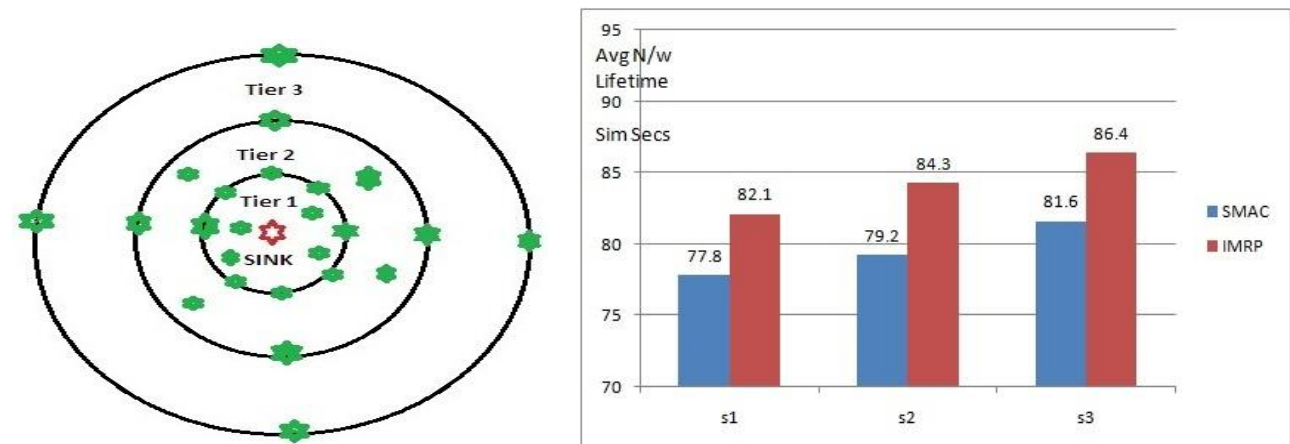

Fig. 6. Scenario 3

Fig. 7. Performance analysis of SMAC and Integrated MAC and Routing (IMAC)

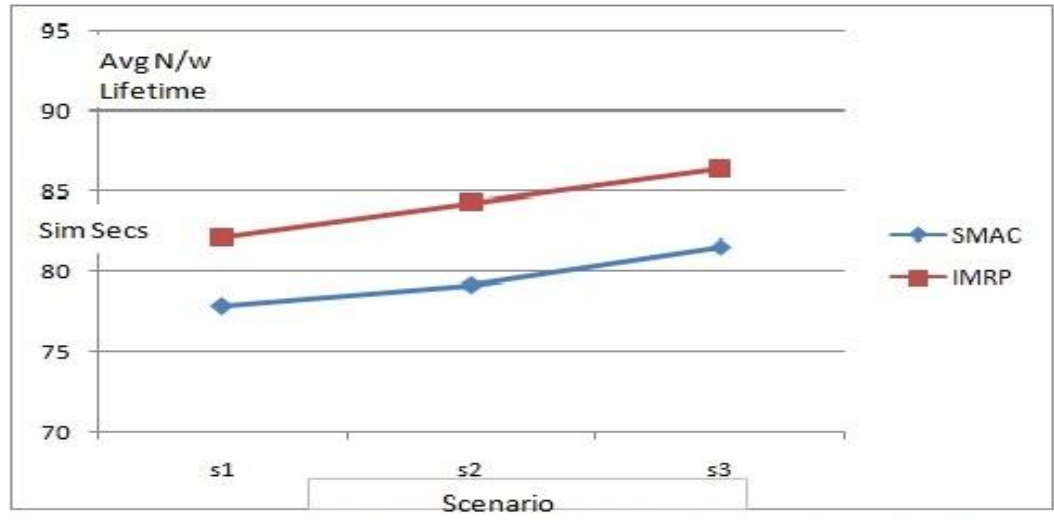

Fig. 8. Performance analysis of SMAC and Integrated MAC and Routing (IMAC)

\section{Conclusion and future scope}

We have addressed the hot-spot problem in WSN and proposed a simple and efficient algorithm to mitigate the hot-spot effect and to improve the overall network life-time. The paper can be further improved by working in the lines of deciding the number of sensors nodes in each tier given a coverage area and for simplicity we have not considered the sleep/wake-up pattern, by considering the sleep wake-up pattern, the effective number of nodes in each tier may still increase but as the sensor nodes are getting cheaper and cheaper it may not be an hindrance. The solution proposed is a trade-off between number of sensor nodes in each tier and the overall life-time of the WSN.

\section{References}

1. Kulkarni, S., A. I y e r, C. R o s e n b e r g. An Address-Light, Integrated MAC and Routing Protocol for Wireless Sensor Networks. - IEEE/ACM Transactions on Networking, Vol. 14, August 2006, No 4, pp. 793-806. 
2. Riva s, H., T. V o i g t, A. D u n kel s. A Simple and Efficient Method to Mitigate the Hot Spot Problem in Wireless Sensor Networks. - IEEE GLOBECOM Wireless Ad Hoc and Sensor Networks.

3. Ye, M., E. Chan, Guih a i. On Mitigating Hot Spots for Clustering Mechanisms in Wireless Sensor Networks. National Laboratory of Novel Software Technology, Nanjing University, China and Department of Computer Science, City University of Hong Kong, China, 1-4244-0507-6/06/\$20.00 C2006 IEEE, pp. 558-561.

4. A b d u 11 a, A. E. A. A., H. N i s h i y a m a, N. K a t o. Extending the Lifetime of Wireless Sensor Networks: A Hybrid Routing Algorithm. - Computer Communications Journal, Vol. 35, May 2012, No 9, pp. 1056-1063.

5. Perillo, M., Z. Cheng, W. Heinzelman. An Analysis of Strategies for Mitigating the Sensor Network Hot Spot Problem. - In: Proc. of Second International Conference on Mobile and Ubiquitous Systems, 2005, pp. 474-478.

6. W ang, J., I. J e an de Di e u, A. D e Le on Diego Jos e, S. L e e, Y.-K. Le e. Prolonging the Lifetime of Wireless Sensor Networks via Hotspot Analysis. - In: Proc. of 10th IEEE/IPSJ International Symposium on Applications and Internet (SAINT), 2010, pp. 383-386.

7. T s e n g, Y u-Ch e e, Y o u-Chi un W an g, K a i-Y a n g Cheng, Y a o-Y u H s i e h. iMouse: An Integrated Mobile Surveillance and Wireless Sensor System. - National Chiao Tung University, Published by the IEEE Computer Society, Vol. 40, 2007, No 6, pp. 60-66. 